\title{
Autonomous Excitation Transfer in Quantum Dot Mixtures via Network of Optical Near-Field Interactions at the Nanoscale
}

\author{
Makoto Naruse ${ }^{* 1,3}$, Ferdinand Peper ${ }^{1}$, Kenji Leibnitz $^{1,2}$, Kouichi Akahane $^{1}$, Naokatsu Yamamoto ${ }^{1}$, \\ Wataru Nomura ${ }^{3}$, Tadashi Kawazoe ${ }^{3}$, Takashi Yatsui ${ }^{3}$, Masayuki Murata ${ }^{2}$, and Motoichi Ohtsu ${ }^{3}$
}

\author{
${ }^{1}$ National Institute of Information \\ and Communications Technology \\ 4-2-1 Koganei, Nukui-kita, \\ Tokyo 184-8749 Japan \\ *E-mail: naruse@nict.go.jp
}

\author{
${ }^{2}$ Department of Information \\ Networking, Graduate School \\ of Information Science and \\ Technology, Osaka University, \\ 1-5 Yamadaoka, Suita \\ Osaka, 565-0871 Japan
}

\author{
${ }^{3}$ Department of Electrical Engineering \\ and Information Systems and the \\ Nanophotonics Research Center, Graduate \\ School of Engineering, The University of \\ Tokyo, 2-11-16 Yayoi, Bunkyo-ku, \\ Tokyo 113-8656, Japan
}

\begin{abstract}
We examine autonomous optical excitation transfer in mixtures of different-sized quantum dots networked via optical near-fields at the nanometer scale. We theoretically and experimentally demonstrate optical excitation transfer via the network of optical near-field interactions among quantum dots. The topology-dependent efficiency of excitation transfer is also investigated. The results of our analysis of autonomous and energy-efficient light-matter interactions at the nanoscale, called nanophotonics, will provide useful insights into the design of robust and energy-efficient information and communications systems and networks.
\end{abstract}

Index Terms-nanophotonics, nanometer-scale systems, nanostructures, network in the nanometer-scale, new generation network, optical excitation transfer, optical near-fields

\section{INTRODUCTION}

$\mathrm{O}$ PTICS is expected to play a crucial role in enhancing system performance to handle the continuously growing amount of digital data and new requirements demanded by industry and society [1]. However, there are some fundamental difficulties impeding the adoption of optical technologies in information processing and communication systems [2-6]. One problem is the poor integrability of optical devices in systems due to the diffraction limit of light. This is because the optical wavelength used in a given system is typically around $1 \mu \mathrm{m}$, which is about 100 times larger than the gate width of present silicon VLSI hardware.

Nanophotonics, on the other hand, which is based on local interactions between nanometer-scale materials via optical near-fields, is not restricted by conventional diffraction of light, allowing ultrahigh-density integration [2-4]. Optical excitation transfer between quantum dots (QDs) via optical near-field interactions will be one of the most important mechanisms in realizing novel devices and systems $[2,3,7,8]$.
Moreover, qualitatively novel features that are unavailable in conventional optics and electronics are enabled by such optical excitation transfer $[9,10]$.

In this paper, we demonstrate optical excitation transfer in a mixture composed of different-sized (larger and smaller) QDs networked via optical near-fields in their vicinities. We theoretically demonstrate an optimal mixture of two differentsized QDs concerning the optical near-field interaction network based on a density matrix formalism, which agrees well with experimental results obtained using CdSe/ZnS QD mixtures with diameters of $2.0 \mathrm{~nm}$ and $2.8 \mathrm{~nm}$. In addition, we theoretically examine the topology-dependent efficiency of optical excitation transfer, which also clearly exhibits autonomous, energy-efficient networking behavior occurring at the nanometer scale.

This paper is organized as follows. In Section II, we describe the physical fundamentals of optical excitation transfer between QDs. In Section III, we discuss a network of optical near-field interactions and analyze the mixturedependent optical excitation transfer theoretically and experimentally. In Section IV, we investigate the topologydependency of the excitation transfer. In Section V, we discuss what we can learn from these physical principles and phenomena physically existing at the nanometer scale and their implications for information and communications applications, as well as information networking.

\section{OPTICAL EXCITATION TRANSFER VIA OPTICAL NEAR- FIELD INTERACTIONS BETWEEN QUANTUM DOTS}

In this section, we first briefly review the fundamental principles of optical excitation transfer involving optical nearfield interactions [2,3]. Conventionally, the interaction Hamiltonian between an electron and an electric field is given 

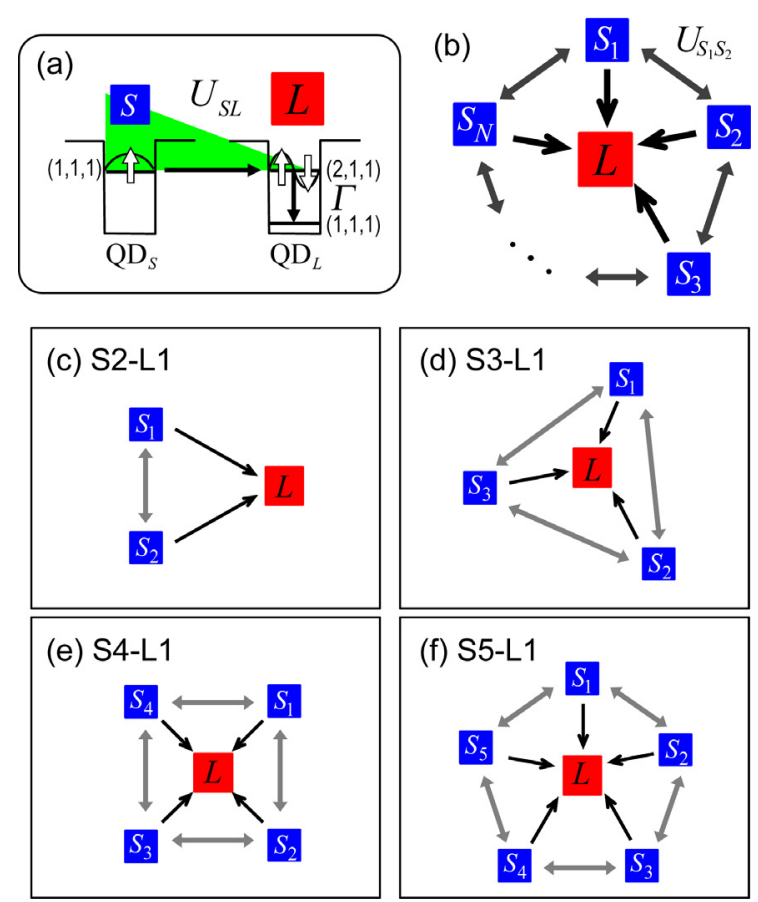

Fig. 1. (a) Optical excitation transfer from smaller quantum dot $\left(\mathrm{QD}_{S}\right)$ to larger one $\left(\mathrm{QD}_{L}\right)$ via optical near-field interactions. (b) Multiple quantum dot system composed of multiple smaller QDs and one larger QD. (c-f) Example systems.

by

$$
\hat{H}_{\mathrm{int}}=-\int \hat{\psi}^{\dagger}(\vec{r}) \vec{\mu} \hat{\psi} \hat{\psi}(\vec{r}) \bullet \hat{\vec{D}}(\vec{r}) d \vec{r}
$$

where $\vec{\mu}$ is the dipole moment, $\hat{\psi}^{\dagger}(\vec{r})$ and $\hat{\psi}(\vec{r})$ are respectively creation and annihilation operators of the electron at $\vec{r}$, and $\hat{\vec{D}}(\vec{r})$ is the operator of electric flux density. In usual light-matter interactions, the operator $\hat{\vec{D}}(\vec{r})$ is a constant since the electric field of propagating light is considered to be constant on the nanometer scale. Therefore, one can derive optical selection rules by calculating a transfer matrix of an electric dipole. As a consequence, in the case of cubic QDs, for instance, transitions to states described by quantum numbers containing an even number are prohibited [2]. In the case of optical near-field interactions, on the other hand, due to the steep electric field of optical near-fields in the vicinity of nanometer-scale matter, an optical transition that violates conventional optical selection rules is allowed $[2,3]$.

Optical excitations in nanostructures, such as QDs, can be transferred to neighboring ones via optical near-field interactions [8-10]. For instance, assume that two cubic QDs whose side lengths are $a$ and $\sqrt{2} a$, which are called $\mathrm{QD}_{S}$ and $\mathrm{QD}_{L}$, respectively, are located close to each other, as shown in Fig. 1(a). Suppose that the energy eigenvalues for the quantized exciton energy level specified by quantum numbers $\left(n_{x}, n_{y}, n_{z}\right)$ in the QD with side length $a\left(\mathrm{QD}_{S}\right)$ are given by

$$
E_{\left(n_{x}, n_{y}, n_{z}\right)}=E_{B}+\frac{\hbar^{2} \pi^{2}}{2 M a^{2}}\left(n_{x}^{2}+n_{y}^{2}+n_{z}^{2}\right)
$$

where $E_{B}$ is the energy of the bulk exciton, and $M$ is the effective mass of the exciton. According to (2), there exists a resonance between the level of quantum number $(1,1,1)$ in $\mathrm{QD}_{S}$ and that of quantum number $(2,1,1)$ in $\mathrm{QD}_{L}$. There is an optical near-field interaction, which is denoted by $U_{S L}$, due to the steep electric field in the vicinity of $\mathrm{QD}_{S}$. It is known that the inter-dot optical near-field interaction is given by a Yukawa-type potential [2]. Therefore, excitons in $\mathrm{QD}_{S}$ can move to the $(2,1,1)$-level in $\mathrm{QD}_{L}$. Note that such a transfer is prohibited for propagating light since the $(2,1,1)$-level in $\mathrm{QD}_{L}$ contains an even number. In $\mathrm{QD}_{L}$, the exciton sees a sublevel energy relaxation, denoted by $\Gamma$, which is faster than the nearfield interaction, and so the exciton goes to the $(1,1,1)$-level in $\mathrm{QD}_{L}$.

In Sections III and IV, we apply these theoretical arguments to systems composed of multiple quantum dots and investigate their impact on fundamental features of optical excitation transfer occurring at the nanometer scale.

(a)

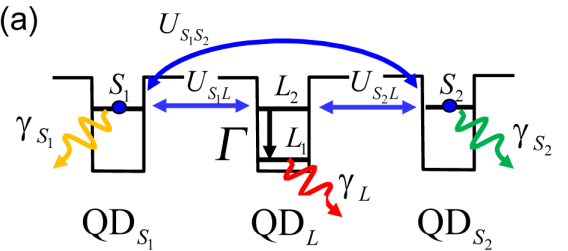

(b)
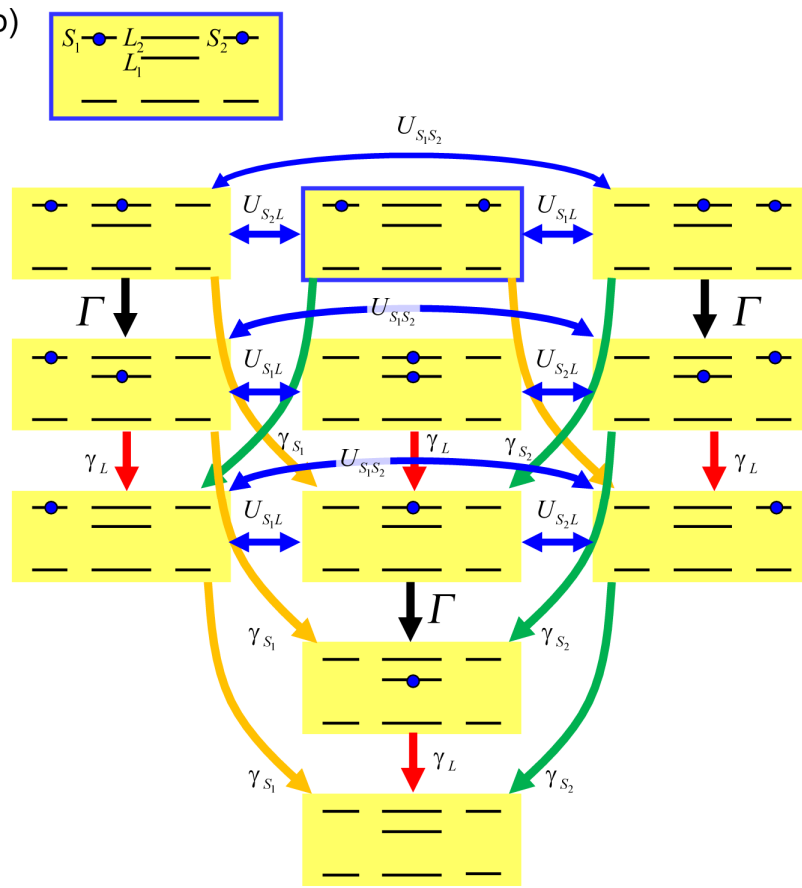

Fig. 2. Example of the system modeling based on a density matrix formalism. (a) Parameterizations for inter-dot near-field interactions, radiative relaxations, and non-radiative relaxations in the $\mathbf{S 2 - L 1}$ system. (b) Schematic representation of state transitions in the $\mathbf{S 2 - L 1}$ system. 


\section{NETWORK OF OPTICAL NEAR-FIELD INTERACTIONS}

Here we consider a quantum dot system shown in Fig. 1(b), where multiple smaller dots (denoted by $S_{i}$ ) can be coupled with one larger dot, denoted by $L$. We also assume inter-dot interactions between adjacent smaller quantum dots; that is, (i) $S_{i}$ interacts with $S_{i+1}(i=1, \ldots, N-1)$ and (ii) $S_{N}$ interacts with $S_{1}$, where $N$ is the number of smaller QDs. For instance the system shown in Fig. 1(c) consists of two smaller QDs and one larger QD, denoted by S2-L1. Similarly, S3-L1, S4-L1, S5-L1 systems are composed of three, four, and five smaller QDs in addition to one large QD, which are respectively shown in Figs. 1(d), (e), and (f).

Now, what is of interest is to maximize the flow of excitations from the smaller dots to the larger one. We deal with this problem theoretically based on a density matrix formalism. In the case of the S2-L1 system, composed of two smaller QDs and one larger QD, the inter-dot interactions between the smaller dots and the larger one are denoted by $U_{S_{i} L}$, and that between the smaller dots is denoted by $U_{S_{1} S_{2}}$, as schematically shown in Fig. 2(a). The radiations from $S_{1}, S_{2}$, and $L$ are respectively represented by the relaxation constants $\gamma_{S_{1}}, \gamma_{S_{2}}$, and $\gamma_{L}$. We suppose that the system initially has two excitations $S_{1}$ and $S_{2}$. With such an initial state, we can prepare a total of eleven bases where zero, one, or two excitation(s) occupy the energy levels; the state transitions are schematically shown in Fig. 2(b). In the numerical calculation, we assume $U_{S_{i} L}=200 \mathrm{ps}$ and $U_{S_{1} S_{2}}=100$ ps as typical parameter values. Following the same procedure, we also
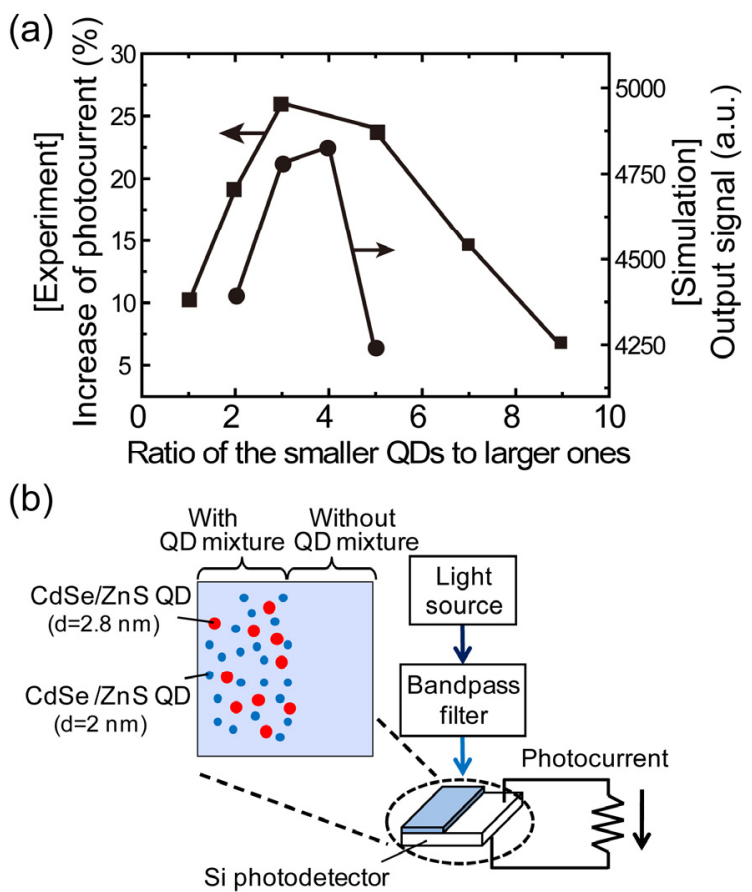

Fig. 3. (a) Optimal ratio of the number of smaller QDs to larger ones so that the optical excitation transfer is the most efficiently induced. (b) Schematic diagram of the experimental demonstration. derive quantum master equations for the S3-L1, S4-L1, and S5-L1 systems with initial states in which all smaller quantum dots have excitations. Finally, we can calculate the population of the lower level of $\mathrm{QD}_{L}$, the time integral of which we regard as the output signal.

We compare the output signal as a function of the ratio of the number of smaller dots to the larger one assuming that the total number of QDs, regardless of their sizes (smaller or larger), in a give unit area is the same. As shown in the circles in Fig. 3(a), the most efficient transfer is obtained when the ratio of the number of smaller dots to the larger one is 4 . That is, increasing the number of smaller quantum dots does not necessarily contribute to increased output signals. Because of the limited radiation lifetime of $\mathrm{QD}_{L}$, not all of the initial excitations can be successfully transferred to $\mathrm{QD}_{L}$ due to the state filling of the lower level of $\mathrm{QD}_{L}$. Therefore, part of the input populations must be decayed at $\mathrm{QD}_{S}$, which results in loss in the transfer from the smaller QDs to the larger one when there are too many excitations in the smaller QDs surrounding one larger QD.

Such an optimal mixture of smaller and larger QDs was experimentally demonstrated by using two kinds of CdSe/ZnS core/shell quantum dots whose diameters were $2.0 \mathrm{~nm}$ and 2.8 $\mathrm{nm}[11,12]$. The quantum dots were dispersed in a matrix composed of toluene and ultraviolet curable resin and were coated on the surface of a silicon photodiode (Hamamatsu Photonics K.K., Si Photodiode S2368). As schematically shown in Fig. 3(b), half of the surface of the photodiode was spin-coated with an ultraviolet-curable resin containing a mixture of quantum dots, which was then cured by ultraviolet radiation, whereas the other half of the surface was coated with the same resin without the quantum dot mixture. Input light was selectively radiated onto each area to evaluate the difference in the generated photocurrent. The increase of the induced photocurrent via the QD-coated area with input light wavelengths between $340 \mathrm{~nm}$ and $360 \mathrm{~nm}$ is shown by the squares in Fig. 3(b). We attribute such an increase to the optical excitation transfer through which the input light wavelength is red-shifted to wavelengths where the photodetector is more sensitive. The maximum increase was obtained when the ratio of the number of smaller QDs to larger ones was $3: 1$. This agrees with the theoretical optimal ratio discussed above.

\section{TOPOLOGY-DEPENDENT, AutonOMOUS, EFFICIENT OPTICAL EXCITATION TRANSFER}

In the previous section, we observe that the amount of optical excitation transferred from the smaller QDs to the larger one depends on the ratio of the number of smaller and larger QDs. This indicates that we could increase the output by further engineering the network structure among the QDs. In this section, by taking the S5-L1 system shown in Fig. 1(f) as an example, we demonstrate that the output signal could be increased when the network among the quantum dots is adequately configured. 

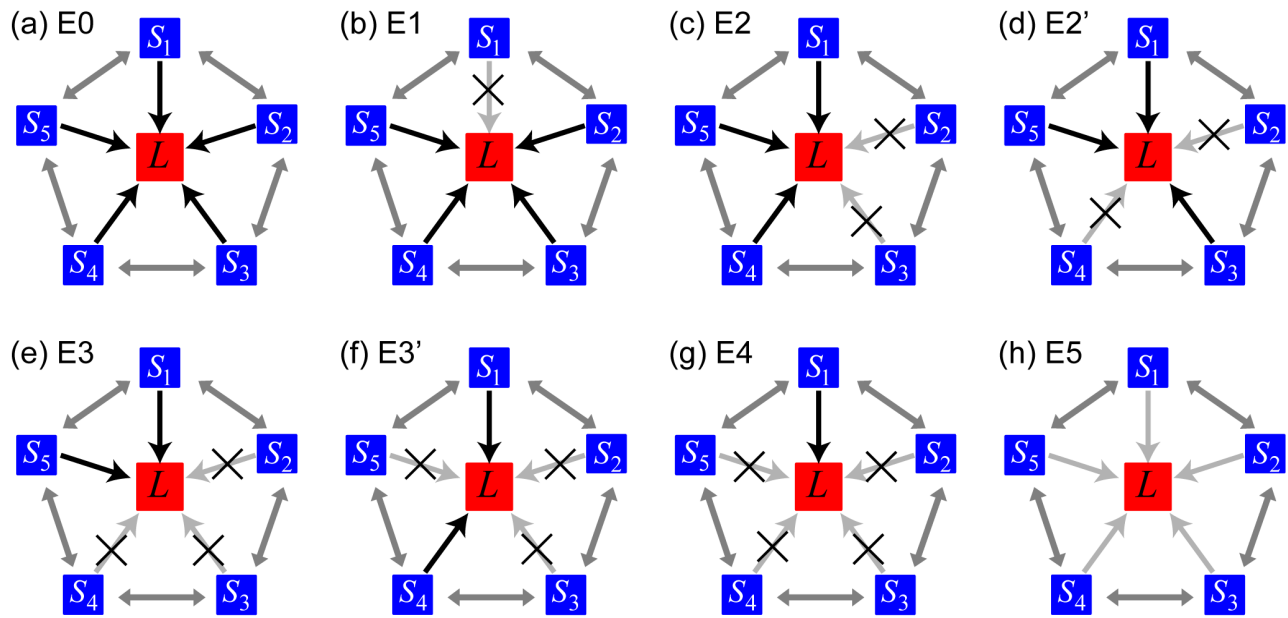

Fig. 4. Eight different network topologies in the S5-L1 system where some of the interactions between $\mathrm{QD}_{S}$ and $\mathrm{QD}_{L}$ are degraded, or lost. (Degraded interactions are indicated by "X")

Figure 4(a), denoted by E0, represents the original S5-L1 system which is the same as the one shown in Fig. 1(f). Here, assume that some of the interactions between $\mathrm{QD}_{S}$ (denoted by $S_{1}$ to $S_{5}$ ) and $\mathrm{QD}_{L}$ are degraded, or lost, due to, for instance, material disorder such as violation of the condition represented by (1). There are in total eight such configurations concerning the symmetry; for instance, when one of the five links between $\mathrm{QD}_{S}$ and $\mathrm{QD}_{L}$ is degraded, such a situation is

(a)

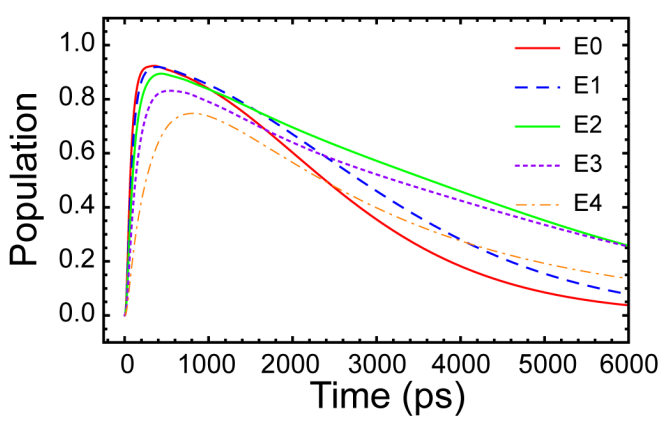

(b)

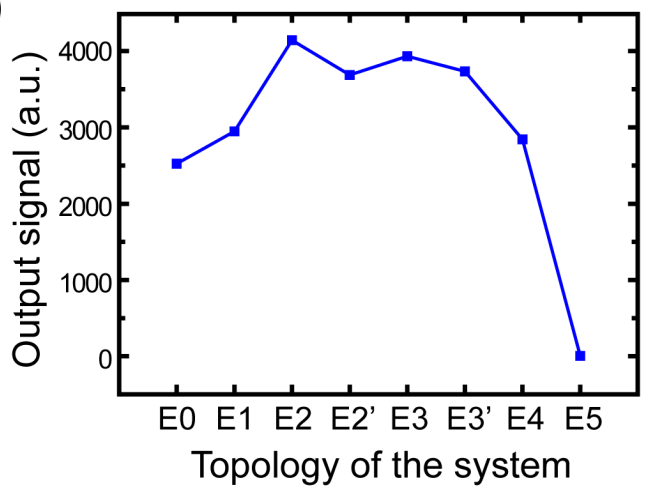

Fig. 5. (a) The time evolution of the populations associated with the QDL in systems E0, E1, E2, E3, and E4 in Fig. 4. (b) Time integrated populations for the systems in Fig. 4, where systems with certain negligible, or essentially nonexistent links result in higher output signal levels. represented by the system $\mathbf{E} 1$ schematically shown in Fig. 4(b). (The interaction between $S_{1}$ and $L$ is indicated by X.) Similarly, when the number of degraded links is two, the system should be represented either by the system E2 or E2' shown respectively in Fig. 4(c) and (d).

Figure 5(a) demonstrates the time evolutions of the populations associated with radiation from $\mathrm{QD}_{L}$. Figure $5(\mathrm{~b})$ summarizes the integrated populations as a function of the network configurations illustrated in Fig. 4. Interestingly, systems with degraded interactions, except the system E5 which has no valid links between $\mathrm{QD}_{S}$ and $\mathrm{QD}_{L}$, exhibit a higher output signal than the system without link errors, namely, the system E0. For example, the system E2 exhibits an output signal about 1.64 times higher than the system E0. This corresponds to the results described in Section III, where the output is maximized when the ratio of the number of $\mathrm{QD}_{S}$ to $\mathrm{QD}_{L}$ is 4 , meaning that the excessively high number of excitations in $\mathrm{QD}_{S}$ connected to $\mathrm{QD}_{L}$ cannot be transferred. Thanks to the "limited" interactions between $\mathrm{QD}_{S}$ and $\mathrm{QD}_{L}$, such as in the case of the systems $\mathbf{E 2}$ and E2', the excitations located in $\mathrm{QD}_{S}$ have more chance to be transferred to $\mathrm{QD}_{L}$. In

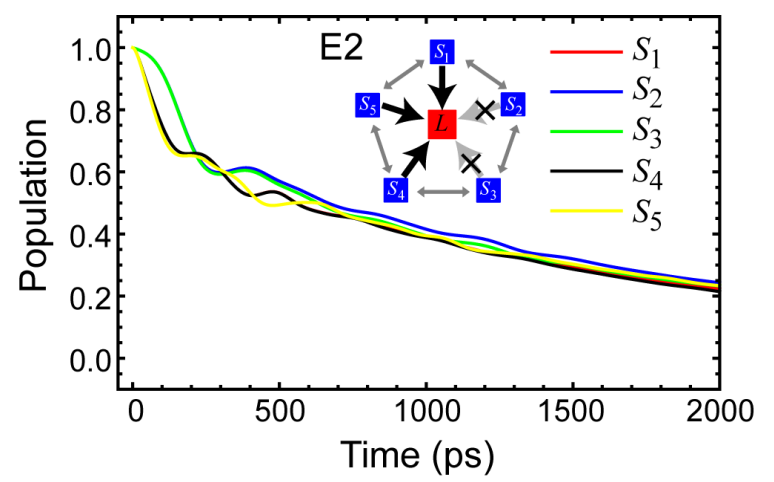

Fig. 6. Time evolutions of the populations associated with the smaller QDs (S1 to S5) in the system E2 in Fig. 4. 
representing such an effect, Fig. 6 summarizes the evolutions of populations associated with $S_{1}$ to $S_{5}$ in the system E2, where the interactions between $\left[S_{2}\right.$ and $\left.L\right]$ and $\left[S_{3}\right.$ and $\left.L\right]$ are negligible, or essentially nonexistent. Note that the populations associated with $S_{2}$ and $S_{3}$ remain at a higher level, indicating that the optical excitations in $S_{2}$ and $S_{3}$ are effectively "waiting" in the smaller dots until they have the opportunity to be transferred to $\mathrm{QD}_{L}$ by way of the interactions between smaller dots.

\section{IMPLICATIONS FOR INFORMATION AND COMMUNICATION SYSTEMS}

Finally, we would like to conclude with a few remarks about how these findings in nanometer-scale light-matter interaction networks can be applied to information and communications technologies (ICTs). The first point we would like to address here is the autonomous behavior of the optical excitations. As we could see, there is no "central controller" in the systems, and yet, efficient transport of the optical excitations is realized, as clearly observed in the discussions above. Such an intrinsic, seemingly intelligent behavior of the nanometer-scale physical system may also provide valuable lessons for designing self-organizing, distributed, complex ICT systems on Internet-scale. In the wake of the current redesign efforts toward a future Internet, novel distributed and autonomous strategies for new generation networks (NwGN) are being considered [13] that are able to cope with the numerous requirements the Internet will be facing in the future. Some of these approaches are based on biological or physical phenomena that exhibit self-* capabilities, e.g., self-organization, self-adaptability, which permit a flexible and autonomous management of the network [14]. Furthermore, using distributed and autonomous network systems helps avoiding unbalanced traffic load and energy consumption [13], as well as reduces the dependence of the network on single points of failure and thus leads to improving the overall sustainability and reliability.

Closely related to this is also the second observation that the increase in the output signal induced by degraded interactions indicates robustness against errors occurring in the system. This point is also of great importance for future communication networks. Since new generation networks are expected to accommodate a large number of heterogeneous end devices, access technologies, network protocols/services, and traffic characteristics, the consideration of failures or sudden fluctuations in performance seems inevitable. Designing robust mechanisms is, therefore, a key issue and utilizing such an intrinsic robustness of nanophotonics, which has the potential to provide superior robustness to errors, while requiring less hardware redundancy than current proposals with redundancies of the order of 10 to 100 [15], may provide helpful guidelines and/or principles for constructing efficient future ICT systems.

Third, it was demonstrated that a single process of optical excitation transfer is about $10^{4}$ times more energy efficient compared with the single bit flip energy required in current electrical devices [16]. A system-level, comprehensive comparison of energy efficiency is an important and timely subject that should be explored in future. These studies will be extremely helpful for developing energy efficient strategies to assist in handling the tremendous growth in traffic and required processing energy anticipated in future communication networks $[17,18]$.

\section{REFERENCES}

[1] K. Sato and H. Hasegawa, "Prospects and Challenges of Multi-Layer Optical Networks," IEICE Trans. Commun., vol. E90-B, pp. 1890-1902, August 2007.

[2] M. Ohtsu, K. Kobayashi, T. Kawazoe, S. Sangu, and T. Yatsui, "Nanophotonics: Design, Fabrication, and Operation of Nanometric Devices Using Optical Near Fields," IEEE J. Sel. Top. Quantum Electron., vol. 8, pp. 839-862, 2002

[3] M. Ohtsu, T. Kawazoe, T. Yatsui, and M. Naruse: "Nanophotonics: Application of Dressed Photons to Novel Photonic Devices, and Systems," IEEE J. Sel. Top. Quantum Electron., vol. 14, pp. 1404-1417, 2008.

[4] H. Hori, "Electronic and Electromagnetic Properties in Nanometer Scales," in Optical and Electronic Process of Nano-Matters, M. Ohtsu, ed., Kluwer Academic, 2001, pp. 1-55.

[5] MONA consortium, A European roadmap for photonics and nanotechnologies, MONA (Merging Optics and Nanotechnologies Association) consortium, 2008.

[6] National Research Council, Nanophotonics: Accessibility and Applicability, National Academies Press, Washington, D.C. , 2008.

[7] T. Unold, K. Mueller, C. Lienau, T. Elsaesser, and A. D. Wieck, "Optical Control of Excitons in a Pair of Quantum Dots Coupled by the Dipole-Dipole Interaction," Phys. Rev. Lett., vol. 94, pp. 137404 1-4, April 2005.

[8] T. A. Klar, T. Franzl, A. L. Rogach, and J. Feldmann, "Super-Efficient Exciton Funneling in Layer-by-Layer Semiconductor Nanocrystal Structures," Adv. Matter., vol. 17, pp. 769-773, March 2005.

[9] M. Naruse, T. Miyazaki, F. Kubota, T. Kawazoe, K. Kobayashi, S. Sangu, and M. Ohtsu, "Nanometric summation architecture based on optical near-field interaction between quantum dots," Opt. Lett., vol. 30, pp. 201-203, January 2005.

[10] M. Naruse, H. Hori, K. Kobayashi, and M. Ohtsu, "Tamper resistance in optical excitation transfer based on optical near-field interactions," Opt. Lett. vol. 32, pp. 1761-1763, June 2007.

[11] M. Naruse, T. Kawazoe, R. Ohta, W. Nomura, and M. Ohtsu, "Optimal mixture of randomly dispersed quantum dots for optical excitation transfer via optical near-field interactions," Phys. Rev. B, vol. 80, pp. 125325 1-7, September 2009.

[12] W. Nomura, T. Yatsui, T. Kawazoe, and M. Ohtsu, "The observation of dissipated optical energy transfer between CdSe quantum dots," $J$. Nanophotonics, vol. 1, pp .011591 1-7, November 2007.

[13] H. Harai, K. Fujikawa, V.P. Kafle, T. Miyazawa, M. Murata, M. Ohnishi, M. Ohta, and T. Umezawa, "Design Guidelines for New Generation Network Architecture," IEICE Trans. Commun., vol. E93-B, no. 3, pp. 462-465, March 2010.

[14] S. Balasubramaniam, D. Botvich, J. Mineraud, W. Donnelly, N. Agoulmine, "BiRSM: bio-inspired resource self-management for all IPnetworks," IEEE Network, vol. 24, no. 3, pp. 20-25, May-June 2010.

[15] A. S. Sadek, K. Nikolić, M. Forshaw, "Parallel information and computation with restitution for noise-tolerant nanoscale logic networks," Nanotechnology, vol. 15, no. 1, pp. 192-210, November 2003.

[16] M. Naruse, H. Hori, K. Kobayashi, P. Holmström, L. Thylén, and M. Ohtsu, "Lower bound of energy dissipation in optical excitation transfer via optical near-field interactions," Opt. Express, vol. 18, pp. A544A553, October 2010.

[17] M. Gupta, S. Singh, "Greening the Internet," in Proc. of ACM SIGCOMM '03, Karlsruhe, Germany, August 2003.

[18] A. Qureshi, R. Weber, H. Balakrishnan, J. Guttag, and B. Maggs. "Cutting the electric bill for internet-scale systems," ACM SIGCOMM Comput. Commun. Rev., vol. 39, no. 4, August 2009. 